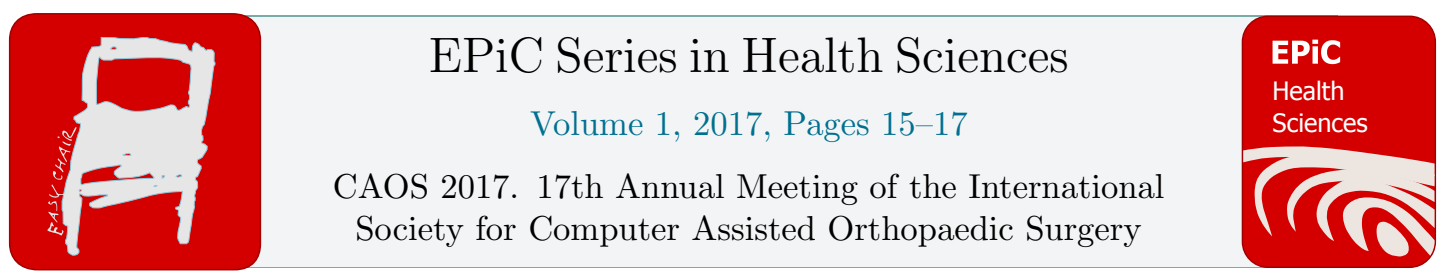

\title{
Learning curve of patient specific templates for TKA. Assessment with a navigation system and the CUSUM test.
}

\author{
Jean-Yves Jenny, Marco De Gori \\ University Hospital Strasbourg, Strasbourg, France \\ jean-yves.jenny@chru-strasbourg.fr
}

\begin{abstract}
The CUSUM analysis allows monitoring the learning curve when introducing PSTs for TKA in an academic department. There may be a significant learning curve to achieve the steady state of accuracy and obtain an acceptable alignment. The decision was taken to discontinue using PSTs for TKA.
\end{abstract}

\section{Introduction}

The patient-specific templates (PST), in which the bone resections are performed with disposable guides fashioned for the specific patient, have been developed with the theoretical advantage of both an outlier rate reduction and a decreased operating time in comparison to conventional instruments during total knee arthroplasty (TKA) (Thienpont 2013), thus leading theoretically to lower costs. There remains controversy about the accuracy of PST in comparison with either navigated or conventional instruments (Barrett 2014). Actually, it has been previously demonstrated that a mismatch between planning and the intraoperative component positioning may occur (Scholes 2014). Furthermore, the learning curve after introducing PST has not been well defined. The goal of the present study was to perform quality control with a commercially available navigation system when introducing PST technique at our academic department. The learning curve during that introduction was assessed by the Cumulative Sum (CUSUM) test. This test allows a sequential quality process control and is well adapted to get an early feedback about surgical performance (Chang 2006). This test may determine if the process is in control, and if some characteristics of the process (such as the mean) deviate from a pre-specified target value. Its use for TKA quality control has been scarcely published (Nizard 2004). We hypothesized that the PST process for TKA was immediately under control at our academic department after its introduction when analyzed with the CUSUM technique. 


\section{Material and methods}

The first 50 TKAs implanted with the use of PST at an academic department were scheduled to enter in a prospective, observational study. Inclusion criteria were a patient with end-stage primary knee osteoarthritis (OA), scheduled for TKA, accepting to undergo a additional preoperative computed tomography (CT) evaluation and accepting to wait 6 weeks for surgery, time required to manufacture the templates. There was no additional exclusion criterion. PST were designed to obtain a $0^{\circ}$ frontal alignment of both femoral and tibia components with respect to the coronal mechanical axes, a $0^{\circ}$ sagittal alignment of the tibia component with respect to the sagittal mechanical axis of the tibia and a 0 to $4^{\circ}$ sagittal alignment of the femoral component with respect to the sagittal mechanical axis of the femur. The height of resection was set at $8 \pm 1 \mathrm{~mm}$ measured from the most distal point of the femoral condyles and the most proximal point of the tibia condyles, and the expected height of resection of the opposite side was calculated.

All TKAs were implanted by an experienced, high volume senior consultant with high experience in knee navigation. PSTs were carefully positioned over the bone and articular surfaces to the best fit position, without any navigated information. Then the 3D femoral and tibia PSTs positioning were recorded. The surgical procedure was then completed following the routine navigated procedure with standard navigated templates.

To assess the $3 \mathrm{D}$ positioning of each template individually and of both templates together as a surrogate of the final TKA positioning, one point was given for each item inside the target, giving a maximal femur and tibia scores of 4 points, and a maximal knee score of 8 points, when all items were fulfilled. Following dataset was used for CUSUM chart plotting: allowable slack $=0.5 \mathrm{SD}$, acceptable limit score $=6$ points for knee score and 2 points for femur and tibia scores. For each measurement Mx, two CUSUMs (upper and lower CUSUMs) were calculated. These sums were plotted against the rank of the observation i. A trend in the process results in a change in the slope of the CUSUM, whereas the values are expected to fluctuate around a horizontal line if the process is in control. The process was considered out of control if upper CUSUM or lower CUSUM is outside the acceptable deviation interval.

\section{Results}

There was no significant difference between the numerical values of femur plan and femur PST positioning for all four items. There was a significant difference between the numerical values of tibia plan and tibia PST positioning for all four items except the sagittal orientation. The knee score was still out of control after the 20th case. Both femur and tibia scores were still out of control after the 20th case as well. The decision was taken to interrupt the study after the 20th case as the learning curve appeared unacceptably long in comparison to the routine navigated technique

\section{Discussion}

The main result of this study is that introduction of PST in an academic center may involve a significant learning curve: the process remained out of control even after 20 procedures. The present results contradict the common belief that introduction of PSTs is easy and does not require special instruction (Hafez 2006). Although learning curves in planning, manufacturing, and validating PSTs have been hypothesized (Thienpont 2013b), no previous study dealing with this question has been published to date. The steady state concerning alignment accuracy when introducing PSTs in a high 
volume center and by an experienced knee surgeon was not obtained after 20 implantations. These results indicate that surgeons should have only a progressive confidence with the self-sitting of PSTs when introducing this technology. Introducing PSTs might involve a significant loss of accuracy, at least when comparing with a navigation controlled implantation. The alignment obtained was significantly different from the planned reconstruction for most of the analyzed items on both femur and tibia sides. However, the present data did not allow explaining this prolonged learning curve.

In conclusion, CUSUM analysis allows monitoring the learning curve when introducing PSTs for TKA in an academic department. There may be a significant learning curve to achieve the steady state of accuracy and obtain an acceptable alignment. The decision was taken to discontinue using PSTs for TKA.

\section{References}

1. Barrett W, Hoeffel D, Dalury D, Mason JB, Murphy J, Himden S. In-vivo alignment comparing patient-specific instrumentation with both conventional and computer assisted surgery (CAS) instrumentation in total knee arthroplasty. J Arthroplasty, 29, 343-347, 2014.

2. Chang WR, McLean IP. CUSUM: a tool for early feedback about performance? BMC Med Res Methodol, 2, 6, 8, 2014.

3. Hafez MA, Chelule KL, Seedhom BB, Sherman KP. Computer-assisted total knee arthroplasty using patient-specific templating. Clin Orthop Relat Res, 444, 184-192, 2006.

4. Nizard RS, Porcher R, Ravaud P, Vangaver E, Hannouche D, Bizot P, et al. Use of the Cusum technique for evaluation of a CT-based navigation system for total knee replacement. Clin Orthop Relat Res, 425, 180-188, 2006.

5. Scholes D, Sahni V, Lustig S, Parker DA, Coolican MRJ. Patient-specific instrumentation for total knee arthroplasty does not match the pre-operative plan as assessed by intra-operative computer-assisted navigation. Knee Surg Sports Traumatol Arthrosc, 22, 660-665, 2014.

6. Thienpont E, Bellemans J, Victor J, Becker R. Alignment in total knee arthroplasty, still more questions than answers...Knee Surg Sports Traumatol Arthrosc, 21, 2191-2193, 2013.

7. Thienpont E, Bellemans J, Delport H, Van Overschelde P, Stuyts B, Brabants K, er al. Patientspecific intruments: industry's innovation with a surgeon's interest. Knee Surg Sports Traumatol Arthrosc, 21, 2227-2233, 2013.

\section{Disclosure}

Jean-Yves JENNY receives royalties from Aesculap, is a paid consultant for Exactech, is an unpaid consultant for FH Orthopedics, is member of the board of the CAOS-International Society and of the French Society for Hip and Knee Surgery 